\title{
Conductas de modificación de la imagen corporal en estudiantes Universitarios Ecuatorianos
}

\section{Modification of the body image behaviours in Ecuadorian University students}

Juan Fernández-Méndez

Universidade da Coruña (España)

Rosa Salamea-Nieto Universidad Técnica de Machala (Ecuador) rsalamea@utmachala.edu.ec

Belén Prieto-Castaño Universidade da Coruña (España) leniprietocastano@yahoo.es

María González Fernández

Universidade da Coruña (España)

Revista Cumbres Vol.4 №2

Versión impresa ISSN 1390-9541

Versión electrónica ISSN 1390-3365

http://investigacion.utmachala.edu.ec/revistas/index.php/Cumbres 


\title{
RESUMEN:
}

Este estudio transversal descriptivo persigue investigar las conductas utilizadas por una población universitaria ecuatoriana de cara a controlar la imagen corporal: empleo de laxantes, vómitos y actividad física. Con esa finalidad se elaboró un protocolo diseñado a tal efecto y se aplicó un instrumento psicométrico de evaluación de la imagen corporal (IMAGEN). Los resultados pusieron de manifiesto que no existían diferencias entre hombres y mujeres en cuanto al modelo de imagen corporal, que la gran mayoría de los participantes en el estudio mostraron puntuaciones bajas en cuanto a su preocupación por la imagen corporal y, por tanto, no se vieron alterados los patrones de alimentación y salud que son propios de los trastornos de la conducta alimentaria como pesarse diariamente, restringir la ingesta de calorías y medidas similares como el empleo de laxantes o los vómitos. De todos los procedimientos empleados para controlar la imagen corporal, el más utilizado por los estudiantes universitarios fue la actividad física.

Palabras clave: Trastornos alimentarios, imagen corporal, actividad física, vómitos y laxantes.

\begin{abstract}
ABSTRAC
This descriptive cross-sectional study aims to investigate behaviors used by an Ecuadorian University population in order to control body image: use of laxatives, vomiting and physical activity. For this purpose was developed a protocol designed for this purpose and applied an assessment of body image (IMAGE) psychometric instrument. The results showed that there were no differences between men and women as model of body image, that the vast majority of participants in the study showed low scores in terms of its concern about body image and, therefore, were not altered patterns of food and health that are specific to the TA as weigh yourself daily restrict the intake of colarías and similar measures such as the use of laxatives or vomiting. Of all the procedures used to control the body image, the most widely used by college students was physical activity.
\end{abstract}

Key words: eating disorders, body image, physical activity, vomiting and laxatives.

\section{INTRODUCCIÓN:}

La alarma social que en los últimos años han despertado los trastornos alimentarios encuentra su justificación por el incremento progresivo y cada vez en edades más tempranas en adolescentes y jóvenes.

Los TCA constituyen un grupo de trastornos psicológicos caracterizados por una conducta alterada ante la ingesta alimentaria (por exceso o por defecto) así 
como la aparición de comportamientos de control de peso. Los sistemas de clasificación CIE-10 (OMS, 1992) y DSM-5 (APA, 2014) incluyen entre los TCA varias categorías entre las que destacan, por sus consecuencias, la anorexia nerviosa (AN) y la bulimia nerviosa (BN).

Buscar la explicación a estos hechos no es fácil, pero sin duda los factores culturales y sociales tienen parte de responsabilidad en que este incremento se produzca. Está demostrado que el constructo "imagen corporal" está involucrado en este trastorno, lo que significa que la insatisfacción y la distorsión de la imagen del propio cuerpo son parcialmente responsables de los trastornos alimentarios.

En el caso de las sociedades occidentalizadas la estética corporal idealizada es la esbeltez para las mujeres y la musculatura para los varones, que son difundidas por poderosas industrias del cine, la moda y medios de comunicación (Mancilla, Vázquez, Díaz, Manuel, Amaya y Álvarez, 2012; Barrientos, Escoto, Bosques, Enríquez, Lugo y Saúl, 2014). A esto se suma la presión familiar para lograr la belleza física, comportamiento que sobresale en dichas culturas, en las que se ha incrementado la preocupación por el peso así como la insatisfacción con el propio cuerpo, lo que se refleja en el aumento de procedimientos destinados a la transformación de la imagen física (Vaquero, Alacid, Muyor y López, 2013).

La imagen corporal supone un constructo multidimensional que representa la manera cómo se ven los individuos a sí mismos; los sentimientos e imágenes que les evoca dicha percepción (Raich, 2000). Un elemento central de la imagen corporal es la actitud hacia el propio cuerpo (Sepúlveda, Botella y León, 2001), definida como evaluación general (positiva o negativa) que las personas realizan de su cuerpo; cuando dicha valoración es negativa se hace más probable la aparición de un TCA. Las actitudes afectan tanto a la conducta (por ejemplo, restricciones alimentarias), los pensamientos (una creencia, por ejemplo, de que un cuerpo delgado es un cuerpo atractivo) como a las emociones (principalmente, culpa o ansiedad) relacionadas con la comida (Gasco, Briñol y Horcajo, 2010), incrementándose este deseo por los ideales estéticos impulsados por el discurso cultural y los medios de comunicación (Alvarenga y Lenz Dunker, 2011). Su aparición se asocia con consecuencias psicosociales negativas que pueden ser precursoras de desajustes psicológicos o comportamientos de evitación o purgativos relacionadas con trastornos de la conducta alimentaria (Cruzat, Haemmerli y García, 2012; Díaz y Cruzat, 2011; Martínez, López-Espinoza, Navarro, López-Uriarte y Salazar, 2014; Martínez, Escoto, Bosques, Ibarra y Juárez, 2015; Rutsztein, Leonardelli, Scappatura, Murawski, Elizathe y Maglio,2013; Vaquero et al., 2013). Una de las principales características de estos trastornos consiste en el miedo a subir de peso y el permanente control en la ingesta de comida, asociado a una excesiva valoración de la imagen corporal. Para modificar la misma, las personas con TCA recurren a conductas que conlleva la reducción del número de calorías ingeridas como la actividad física, vómitos o laxantes.

El objetivo del presente trabajo consistía en valorar las conductas relacionadas con la modificación de la imagen corporal en una muestra de participantes universitarios de Ecuador: vómitos, empleo de laxantes y ejercicio físico realizado en los últimos meses. 


\section{MATERIALES Y MÉTODOS}

\section{Participantes}

Participaron un total de 1023 estudiantes (59,6\% mujeres y 40,4\% varones) con un rango de edad de 16 a 45 años (media de 22,48 y desviación típica de 3,86$)$. La muestra global fue reclutada entre individuos que estaban cursando estudios en ese momento en cinco facultades públicas de la Universidad de Machala (Ecuador). En la Tabla 1 se refleja la frecuencia y porcentaje de participantes de cada una de las facultades.

\begin{tabular}{|c|l|l|} 
Tabla 1. Facultades de las que provenían los participantes \\
\hline & FRECUENCIA & PORCENTAJE \\
\hline UACS & 218 & 21,3 \\
UACQS & 193 & 18,9 \\
UACA & 108 & 10,6 \\
UAIC & 104 & 10,2 \\
Total & 1023 & 100,0
\end{tabular}

El muestreo fue no probabilístico, utilizando las facultades como procedimiento para respetar el agrupamiento natural de los estudiantes. Se utilizó un diseño expostfacto simple de corte transversal que permitió la comparación entre grupos.

Instrumentos

Para llevar a cabo este estudio transversal se han aplicado dos instrumentos, uno de elaboración propia, y otro, que fue una prueba psicométrica de fiabilidad y validez demostrada en el contexto de los trastornos alimentarios: IMAGEN.

El Cuestionario sobre conductas hacia la imagen corporal permite la evaluación de comportamientos que pueden modificar la imagen corporal, y fue desarrollado a tal efecto por los miembros de la investigación. El cuestionario se estructura en tres partes: datos socio-biográficos, historia personal, y conductas de control de la ingesta y del peso.

Evaluación de la insatisfacción de la Imagen Corporal -IMAGEN(Solano y Cano, 2010). Es un instrumento de evaluación de fácil y rápida aplicación, que mide la insatisfacción con la imagen corporal. Su objetivo es la detección temprana de un TCA. Consta de 38 ítems y permite la obtención de una puntuación total de insatisfacción con la imagen corporal, y puntuaciones en sus principales componentes: escala de insatisfacción cognitivo-emocional, que hace referencia al deseo y necesidad de tener un cuerpo delgado o realizar una dieta, al miedo a engordar, la sensación de estar ganando peso o la irritabilidad y el malestar emocional asociados al aspecto físico; escala de insatisfacción perceptiva, que considera la creencia o sensación subjetiva de haber alcanzado un peso por encima del deseado o una figura deforme y, por último, escala de insatisfacción comportamental, que analiza aspectos relacionados con la 
producción del vómito, el uso de laxantes, el hecho de tirar comida, realizar ejercicio físico de manera excesiva, etc.

\section{Procedimiento}

Los participantes que decidieron colaborar voluntariamente, tras consentimiento informado, cumplimentaron los cuestionarios en grupo, bajo la supervisión de colaboradores debidamente adiestrados y cualificados para la aplicación de dichas pruebas. Dichos colaboradores, entre otros aspectos, se encargaban de informar acerca de los objetivos de la presente investigación y de la manera en que debían rellenar los distintos autoinformes. Para tomar contacto con los estudiantes se pidió permiso a los órganos de gobierno de las distintas unidades académicas. Los estudiantes universitarios recibieron los cuestionarios a lo largo del horario lectivo al inicio de las clases. Antes de cubrir el cuestionario, fueron informados acerca de la confidencialidad del tratamiento de los datos en consonancia con las distintas leyes de protección de datos.

\section{RESULTADOS}

El peso mínimo de la muestra se sitúa en 35 kilogramos y el máximo en 150, siendo el promedio de 64, 76 (DT = 14,15); por otro lado, la estatura oscila entre 125 centímetros y 150, con estatura media de 163 (DT =0,09). La obsesión media por la delgadez de los participantes fue de 10,13 (DT = 7,31), valorada en una escala que oscilaba entre $0 \mathrm{y}$ 34, significando el "0" ninguna obsesión y el "34" la máxima obsesión. En la Tabla 2 se muestra el número de episodios de vómitos, así como el empleo de laxantes y el ejercicio

Tabla 2. Conductas de modificación de la imagen corporal

\begin{tabular}{|l|l|l|}
\hline & MEDIA & DESV. TÍP. \\
\hline VÓMITOS & 0,10 & 0,47 \\
\hline LAXANTES & 0,14 & 0,54 \\
\hline EJERCICIO FÍSICO & 1,97 & 1,71
\end{tabular}
físico realizado en los últimos tres meses.

El número medio de episodios relacionados con la provocación del vómito para controlar el peso en los últimos tres meses fue bajo, resultando muy similar la tasa promedio con la que los sujetos habían utilizado laxantes para regular el peso en el último trimestre. Por otra parte, la frecuencia con la que realizaron 60 minutos o más de ejercicio físico también durante los tres últimos meses fue, aproximadamente, de dos veces. Se aplicó la t de Student y no se encontraron diferencias estadísticamente significativas entre varones y mujeres en cuanto a la utilización de estas conductas, la satisfacción con la imagen corporal y la obsesión por la delgadez.

La mera inspección visual de la Figura 1 permite apreciar las puntuaciones obtenidas por los estudiantes en cada una de las conductas realizadas para reducir el número de calorías ingeridas y bajar de peso, modificando de esta manera la imagen corporal. Es muy alto el número de participantes que no se han provocado el vómito, resultando bajo el porcentaje de quienes han realizado dicha conducta una vez o más a la semana: 1,1\%. También es muy elevado el número de participantes que no han usado laxantes para 
adelgazar, y muy bajo el de quienes los han usado una vez o más a la semana $(1,3 \%)$. Finalmente, la conducta a la que más ha recurrido la muestra de participantes con la finalidad de modificar la imagen corporal es la actividad física, que ha sido realizada una o más veces al día en el caso del 37,3\% de los participantes.

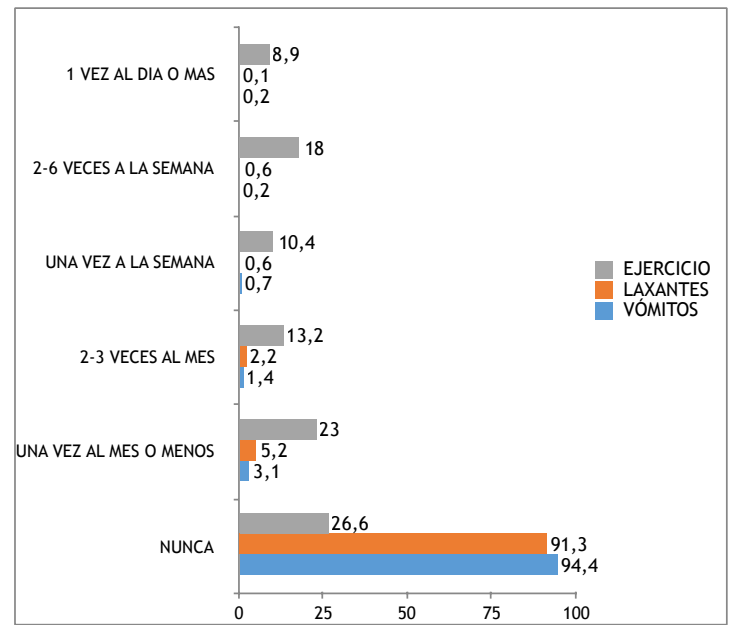

Figura 1. Porcentaje en cada una de las variables en la muestra de participantes

En la Tabla 3 se ofrece la frecuencia y el porcentaje de conductas llevadas a cabo por los estudiantes de cada una de las unidades académicas de la Universidad de Machala que han participado en este estudio. Se puede observar que la distribución de frecuencias y porcentajes es muy similar entre estudiantes de las distintas unidades en cuanto a la provocación del vómito y al uso de laxantes; sin embargo, existen diferencias (aunque no estadísticamente significativas) en cuanto al ejercicio físico realizado semanalmente y diariamente, mostrando la UACA y la UAIC valores superiores. Como consecuencia de estas conductas, un total de 125 personas $(12,22 \%)$ han perdido 9 kilogramos de peso o más en los últimos meses. Además, de la disminución de la ingesta de calorías, llevada a cabo por el 13,14\% de los participantes.

Tras la aplicación de la prueba de correlación de Pearson, se encontraron asociaciones positivas entre el valor global en la insatisfacción corporal y en ciertas variables de la prueba IMAGEN, más concretamente, la insatisfacción cognitivo-emocional $(r x y=0,687 ; p<0,01)$ y la insatisfacción perceptiva $(r \times y=0,557 ; p<0,01)$.

Finalmente, para verificar la existencia o no de diferencias significativas entre cada variable considerada en la prueba IMAGEN y en las diferentes unidades académicas, se empleó un ANOVA (análisis de varianza). En el caso concreto, la aplicación de la prueba mostró que no existían diferencias entre las unidades académicas en las medidas de insatisfacción con la imagen corporal así como en los principales componentes (insatisfacción cognitivo-emocional, perceptiva y comportamental). 
Tabla 3. Frecuencia y porcentaje de provocación de vómitos en cada una de las Unidades Académicas

\begin{tabular}{|c|c|c|c|c|c|c|c|}
\hline & & $\begin{array}{l}\text { UACE } \\
\text { N (\%) }\end{array}$ & $\begin{array}{l}\text { UACS } \\
\text { N (\%) }\end{array}$ & $\begin{array}{c}\text { UACQS } \\
\text { N (\%) }\end{array}$ & $\begin{array}{l}\text { UACA } \\
\text { N (\%) }\end{array}$ & $\begin{array}{l}\text { UAIC } \\
\text { N (\%) }\end{array}$ & TOTAL \\
\hline \multirow{6}{*}{ 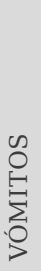 } & NUNCA & $377(94,25)$ & $207(94,95)$ & $181(93,78)$ & $102(94,44)$ & $99(95,19)$ & 966 \\
\hline & UNA VEZ AL MES O MENOS & $10(2,5)$ & $8(3,67)$ & $8(4,14)$ & $4(3,70)$ & $2(1,92)$ & 32 \\
\hline & 2-3 VECES AL MES & $6(1,5)$ & $2(0,92)$ & $3(1,55)$ & $1(0,93)$ & $2(1,92)$ & 14 \\
\hline & UNA VEZ A LA SEMANA & $5(1,25)$ & $0(0)$ & $0(0)$ & $1(0,93)$ & $1(1,92)$ & 7 \\
\hline & 2-6 VECES A LA SEMANA & $1(0,25)$ & $0(0)$ & $1(0,52)$ & $0(0)$ & $0(0)$ & 2 \\
\hline & 1 VEZ AL DIA O MAS & $1(0,25)$ & $1(0,46)$ & $0(0)$ & $0(0)$ & $0(0)$ & 2 \\
\hline \multirow{6}{*}{ 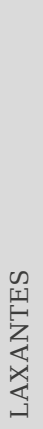 } & NUNCA & $365(91,25)$ & $189(86,70)$ & $177(91,71)$ & $101(93,52)$ & $102(98,07)$ & 934 \\
\hline & UNA VEZ AL MES O MENOS & $22(5,5)$ & $18(4,50)$ & $6(3,10)$ & $6(5,55)$ & $1(0,96)$ & 53 \\
\hline & 2-3 VECES AL MES & $9(2,25)$ & $7(1,75)$ & $5(2,59)$ & $1(0,92)$ & $1(0,96)$ & 23 \\
\hline & UNA VEZ A LA SEMANA & $2(0,5)$ & $2(94,25)$ & $2(1,03)$ & $0(0)$ & $0(0)$ & 6 \\
\hline & 2-6 VECES A LA SEMANA & $1(0,25)$ & $2(94,25)$ & $3(1,55)$ & $0(0)$ & $0(0)$ & 6 \\
\hline & 1 VEZ AL DIA O MAS & $1(0,25)$ & $0(0)$ & $0(0)$ & $0(0)$ & $0(0)$ & 1 \\
\hline \multirow{6}{*}{ 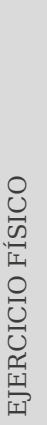 } & NUNCA & $123(30,75)$ & $68(31,19)$ & $47(24,35)$ & $16(14,81)$ & $18(17,31)$ & 272 \\
\hline & UNA VEZ AL MES O MENOS & $90(22,50)$ & $56(25,69)$ & $49(25,38)$ & $20(18,51)$ & $20(19,23)$ & 235 \\
\hline & 2-3 VECES AL MES & $44(11,00)$ & $23(10,55)$ & $34(17,61)$ & $18(16,66)$ & $16(15,38)$ & 135 \\
\hline & UNA VEZ A LA SEMANA & $41(10,25)$ & $22(10,09)$ & $16(8,29)$ & $13(12,04)$ & $14(13,46)$ & 106 \\
\hline & 2-6 VECES A LA SEMANA & $69(17,25)$ & $32(14,67)$ & $32(16,58)$ & $26(24,07)$ & $25(24,04)$ & 184 \\
\hline & 1 VEZ AL DIA O MAS & $33(8,25)$ & $17(7,79)$ & $15(7,77)$ & $15(13,88)$ & $11(10,57)$ & 91 \\
\hline
\end{tabular}

\section{DISCUSIÓN Y CONCLUSIONES}

El presente estudio de naturaleza transversal es relevante porque deja entrever las relaciones entre el modelo de imagen corporal y el de ciertas conductas de modificación de la misma, en una amplia muestra de estudiantes universitarios ecuatorianos.

La mayoría de los participantes en el estudio mostraron puntuaciones bajas en cuanto a su preocupación por la imagen corporal y, por tanto, no se vieron alterados los patrones de alimentación y salud que son propios de los TCA como el hecho de pesarse diariamente, restringir la ingesta de calorías y otras medidas similares como el empleo de laxantes o la provocación de vómitos. Además, se hace evidente que no existen diferencias estadísticamente significativas entre las distintas unidades académicas en cuanto a la imagen 
corporal global y los distintos componentes de la misma tal y como son considerados con la prueba IMAGEN. Estos datos son similares a otros que se han encontrado en países de la Unión Europea como España (Mosquera, Fernández y Mosquera, 2014; Rivas, Bersabé, y Castro, 2001).

Llama poderosamente la atención, que no existan diferencias significativas entre varones y mujeres en cuanto a la satisfacción con la imagen corporal, la obsesión por la delgadez y las conductas de control de la imagen corporal. Por tanto, no se confirma la hipótesis que señala que las mujeres conceden mayor importancia a su imagen corporal (Montero, Morales y Carbajal, 2004; Mosquera et al., 2014). Por lo tanto, a diferencia de trabajos realizados en países de la Unión Europea o los Estados Unidos no se aprecian diferencias en la percepción de la imagen corporal mediante modelos en función del género. López, Prado, Montilla, Molina, Da Silva y Arteaga (2008) referenciando Furnham, Badmin y Sneade (2002).

En futuras líneas de investigación deberá ponerse de manifiesto si las mujeres ecuatorianas siguen un modelo de imagen corporal diferente al de mujeres de países europeos o estadounidenses.

\section{REFERENCIAS BIBLIOGRÁFICAS:}

Alvarenga, M. dos S., \& Lenz Dunker, K. L. (2011). Influencia de los medios de comunicación e insatisfacción corporal en estudiantes brasileñas. Revista Mexicana de Trastornos Alimentarios, 2, 20-28

American Psychiatric Association (2014). DSM-5. Manual Diagnóstico y Estadístico de los Trastornos Mentales. Barcelona: Panamericana.

Barrientos Martínez, N., Escoto, C., Bosques, L. E., Enríquez Ibarra, J., Lugo, J., \& Saúl, C. (2014). Interiorización de ideales estéticos y preocupación corporal en hombres y mujeres usuarios de gimnasio. Revista mexicana de trastornos alimentarios, 5(1), 29-38.

Cruzat, C., Haemmerli, C., \& García, A. (2012). Trastornos de la conducta alimentaria: Reflexionando en torno a las variables socioculturales. Revista de Trastornos Alimentarios, 54-61.

Díaz, F., \& Cruzat, C. (2011). Revista Mexicana de Trastornos Alimentarios Mexican Journal of Eating Disorders. Revista Mexicana de Trasntornos Alimentarios, 2, 1-9.

Furnham, Badmin y Sneade (2002). Body image dissatisfaction: gender differences in eating attitudes, self-esteem, and reasons for exercise. Department of Psychology, University College London.

Gasco, M., Briñol, P., y Horcajo, J. (2010). Cambio de actitudes hacia la imagen corporal: el efecto de la elaboración sobre la fuerza de las actitudes. Psicothema, 22, 71-76.

López, P., Prado P., J. R., Montilla, M., Molina, Z., Da Silva, G. y Arteaga, F. (2008). Insatisfacción por la imagen corporal y la baja autoestima por la apariencia física en estudiantes de la Facultad de Medicina de la Universidad de los Andes del Estado Mérida, Venezuela. Revista Movimiento Humano y Salud, 5(1), 1-14.CIE-10 (OMS, 1992) 
Mancilla, A., Vázquez Arévalo, R., Díaz, M., Manuel, J., Amaya Hernández, A., \& Álvarez Rayón, G. (2012). Insatisfacción corporal en niños y preadolescentes: Una revisión sistemática. Revista mexicana de trastornos alimentarios, 3(1), 62-79.

Martínez, A., López-Espinoza, A., Navarro, M., López-Uriarte, P., \& Salazar, J. (2014). Trastornos de la conducta de beber: una propuesta de investigación. Revista Mexicana de Trastornos Alimentarios, 5, 58-69.

Martínez, N., Escoto, C., Bosques, L., Ibarra, J., \& Juárez, C. (2015). Interiorización de ideales estéticos y preocupación corporal en hombres y mujeres usuarios de gimnasio. Revista Mexicana de Trastornos Alimentarios, 5(1), 29-38.

Montero, P., Morales, E. M., Carbajal, A. (2004). Valoración de la percepción de la imagen corporal mediante modelos anatómicos. Antropo, 8, 107-116.

Mosquera, S. y Fernández, J.C., y Mosquera, A. (2014). Estudio exploratorio sobre factores de riesgos en el desarrollo de trastornos alimentarios. La obsesión por la imagen corporal. Barcelona: Editorial Académica Española.

OMS (1992). CIE-10. Trastornos mentales y del comportamiento. Madrid: Meditor. Trasntornos Alimentarios, 4, 45-57.

Raich, R.M. (2000). Imagen corporal. Madrid. Pirámide.

Rivas, T., Bersabé, R. y Castro, S. (2001). Prevalencia de los trastornos de la conducta alimentaria en Málaga (España). Salud Mental, 24, 25-31.

Rutsztein, G., Leonardelli, E., Scappatura, M. L., Murawski, B., Elizathe, L., \& Maglio, A. (2013). Psychometric properties of the Eating Disorders Inventory-3 (EDI-3) among female adolescents from Argentina. Revista Mexicana de Trastornos Alimentarios., 4, 1-14.

Sepúlveda, A. R., Botella, J. y L eón, J. A. (2001). L a alteración de la imagen corporal en los trastornos de la alimentación: un meta-análisis. Psicothema,13, 7-16.

Solano N. y Cano A. (2010). Imagen. Evaluación de la insatisfacción con la imagen corporal. Madrid: Editorial TEA

Vaquero, R., Alacid, F., Muyor, J., \& López, P. (2013). Imagen corporal; revisión bibliográfica. Nutrición Hospitalaria, 27-35. 
